\title{
Efecto de los elementos menores en la calidad molinera del arroz (Oryza sativa L.) variedad F-2000
}

\author{
Effect of Minor Elements on the Milling Quality \\ of F-2000 Rice Variety (Oryza sativa L.)
}

\section{José Alirio Hernández Quiñónez ${ }^{\mathrm{ac}}$, Seir Antonio Salazar Mercado ${ }^{\text {bd }}$ y Edgar Alfonso Rodríguez Araújo ${ }^{\text {ae }}$}

\footnotetext{
a Departamento de Agronomía, Facultad de Ciencias Agrarias y del Ambiente, Universidad Francisco de Paula Santander, Cúcuta, Colombia

${ }^{\text {b }}$ Departamento de Biología, Facultad de Ciencias Básicas, Universidad Francisco de Paula Santander, Cúcuta, Colombia

' https://orcid.org/0000-0002-5177-962X

${ }^{\mathrm{d}}$ http://orcid.org/0000-0002-3287-703X

e edgarrodriguez@ufps.edu.co | https://orcid.org/0000-0003-3868-1705
}

\section{RESUMEN}

Esta investigación evaluó la aplicación de fertilizantes que contenían elementos menores como mejoradores de la calidad molinera del arroz (Oryza sativa L.) en un suelo clasificado como Typic Xerofluvents. Se valoró un área de 2,5 ha, divididas en cuatro piscinas cultivadas con arroz variedad Fedearroz 2000. Se utilizó un diseño de bloques completos al azar con cinco tratamientos y cuatro repeticiones. Las variables de producción evaluadas fueron: densidad de plantas $\left(46,27 \pm 320\right.$ plantas $\left./ \mathrm{m}^{2}\right)$, macollamiento $\left(59,48 \pm 821,26\right.$ macollas $\left./ \mathrm{m}^{2}\right)$, número panículas/ área $\left(8,53 \pm 440\right.$ panículas $\left./ \mathrm{m}^{2}\right)$, número espiguillas/panícula $(2,76 \pm 12,3$ espiguillas/panícula), número de granos totales $(15,44 \pm 138,77$ granos), número de granos llenos $(7,88 \pm 116$ granos) y número de granos vanos $(19,88 \pm$

22,28 granos). Para evaluar el componente de moline-

Citation: Hernández-Quiñónez, J. A., Salazar-Mercado, S. A.y Rodríguez-Araújo, E. A. (2021). Efecto de los elementos menores en la calidad molinera del arroz (ORYZA SATVA L.) variedad F-2000. Mutis, 11(1), 8-21. doi: https://doi.org/10.21789/22561498.1711

Recibido: Diciembre 7, 2020. Aceptado: Diciembre 30, 2020.

Copyright: $\odot 2021$. Hernández-Quiñónez, J. A., Salazar-Mercado, S. A. y Rodríguez-Araújo, E. A. (2021). This is an open-access article, which permits unrestricted use, distributions and reproduction in any medium, provided the original author and source are credited.

Competing Interests: The authors have no conflict of interest. ría se utilizaron 500 gramos de granos y se valoraron las siguientes variables: porcentaje de vaneamiento $(15,24 \pm 18,48)$, masa blanca $(70,57 \pm 70,68)$, grano entero $(446,9 \pm 59,65)$, cristal $(176,93 \pm 25,24 \%)$, harina $(79 \pm 12,82)$, cáscara $(7,89 \pm 20,85)$ y grano yesado $(82,08 \pm 2,08)$. Para determinar el rendimiento $(37,19$ $\pm 7.689 \mathrm{Kg} / \mathrm{ha}$ ) se cosechó y se pesó cada tratamiento por separado. Se presentaron diferencias significativas $(p<0,0001)$ entre los tratamientos, siendo T2 (fertilización tradicional de NPK + fertilización edáfica con elementos menores) el que presentó mejor comportamiento entre variables de producción, mientras que 
T5 (fertilización tradicional de NPK + fertilización foliar con elementos menores + fertilización foliar con silicio) reportó las mejores variables de molinería. La fertilización con elementos menores contribuye a mejorar la calidad molinera y el rendimiento del arroz, puesto que fortalece el sistema inmune de la planta, combate agentes bióticos y evita los granos vacíos.

Palabras clave: fertilización edáfica, fertilización foliar, macolla, panícula, grano entero.

\section{ABSTRACT}

The research evaluated the application of fertilizers containing minor elements as milling quality improvers of rice (Oryza sativa $\mathrm{L}$ ) in Typic Xerofluvents soils. We assessed an area of 2.5 ha divided into four ponds and cultivated with Fedearroz 2000 rice variety. A randomized complete block design with five treatments and four replications was used. The production variables evaluated were: plant density $\left(46.27 \pm 320\right.$ plants $\left./ \mathrm{m}^{2}\right)$, tillering $(59.48$ \pm 821.26 tillers $\left./ \mathrm{m}^{2}\right)$, number of panicles/area $\left(8.53 \pm 440\right.$ panicles $\left./ \mathrm{m}^{2}\right)$, number of spikelets/panicle $(2.76 \pm 12.3$ spikelets/panicle), number of total grains (15.44 \pm 138.77 grains), number of filled grains ( $7.88 \pm 116$ grains), and number of vain grains (19.88 \pm 22.28 grains). 500 grams of kernels were used to evaluate the milling component, assessing the following variables: percentage of spike sterility $(15.24 \pm 18.48)$, white mass $(70.57 \pm 70.68)$, whole grain $(446.9 \pm 59.65)$, crystal $(176.93 \pm 25.24 \%)$, flour $(79 \pm 12.82)$, husk $(7.89 \pm 20.85)$, and plaster grain $(82.08$ $\pm 2.08)$. Each treatment was harvested and weighed separately in order to determine yield $(37.19 \pm 7,689 \mathrm{~kg} /$ ha). There were significant differences $(p<0.0001)$ among the treatments, with T2 (traditional NPK fertilization + soil fertilization with minor elements) showing the best performance among production variables and T5 among milling variables (traditional NPK fertilization + foliar fertilization with minor elements + foliar fertilization with silicon). Fertilization with minor elements improves milling quality and rice yield by strengthening the plant's immune system, thus combating biotic agents and avoiding empty grains.

Keywords: Soil fertilization, foliar fertilization, bunch, panicle, whole grain.

\section{INTRODUCCIÓN}

El arroz es una planta liliopsida que pertenece a la familia de las gramíneas. Existen 19 especies, siendo el arroz común (Oryza sativa L.) la más importante, puesto que es un alimento básico de la canasta familiar para más de la mitad de la población mundial y el segundo cereal con mayor área cosechada. Su producción promedio anual alcanza, aproximadamente, 650 millones de toneladas (Barona, 2010; HernándezRodríguez et al., 2014; Kato \& Katsura, 2014; Rives et al., 2007). Más del $85 \%$ del área mundial plantada con arroz se riega, principalmente por inundaciones, porque este método aumenta los rendimientos (Botta et al., 2015).

La calidad molinera se define a partir de la cantidad de grano que permanece entero o en tres cuartos de su tamaño después de ser sometido al proceso de descascarado y pulido. La calificación del arroz molinado en términos de rendimiento en molino y calidad no depende únicamente del tipo o condición del equipo o de la destreza del operario, sino que está determinada en gran parte por la calidad del arroz en cáscara (Gnanamanickam, 2009; Mohanty, 2013; Muthayya et al., 2014). El desempeño de este producto durante la molienda está influenciado en gran medida por las características del lote de arroz, incluyendo factores genéticos y ambientales, condiciones de procesamiento y el tipo de molino utilizado (FAO, 2009, 2014).

Entre los factores que influyen en la calidad de molienda se encuentra la disponibilidad de nutrientes en el suelo, particularidad que define un buen lote de arroz. Sin embargo, la mayoría de los suelos presentan deficiencias de ciertos elementos o en ocasiones estos no se encuentran disponibles, lo que hace necesaria la aplicación de fertilizantes para suplir 
requerimientos de tipo nutricional (Dutta et al., 2016; Fairhurst \& Witt, 2002; Serraj et al., 2011).

El grupo de nutrientes se encuentra conformado por nitrógeno $(\mathrm{N})$, fósforo $(\mathrm{P})$ y potasio $(\mathrm{K})$, denominados macro nutrientes primarios, seguidos de azufre (S), calcio (Ca) y magnesio (Mg), nombrados como macronutrientes secundarios (Alshaal \& El-Ramady, 2017; Cakmak \& Kutman, 2017; IFPRI, 2014). Lo anterior se ha determinado según la cantidad de cada nutriente utilizada por la planta y la frecuencia con que en la práctica es necesaria su aportación al cultivo. No obstante, existen elementos que son absorbidos en mínimas cantidades, pero que desempeñan funciones fisiológicas muy importantes. Estos son los elementos menores hierro $(\mathrm{Fe})$, cobre $(\mathrm{Cu})$, zinc $(\mathrm{Zn})$, manganeso $(\mathrm{Mn})$, molibdeno (Mo), boro (B) y silicio (Si), en el caso particular del arroz (Bashir et al., 2013; Das et al., 2013; Virgili, 1996; Zhang et al., 2017).

Se ha comprobado que la aplicación de elementos menores incrementa la producción de arroz en porcentajes significativos debido a que estos forman parte de enzimas que ayudan a la asimilación de los demás elementos, lo cual se traduce en un mayor aprovechamiento de los nutrientes por parte de la planta. Sin embargo, los estudios realizados en campo solo se limitan a la parte productiva (Alamdari \& Mobasser, 2014; Álvarez et al., 2008; Bouis \& Saltzman, 2017; Meena \& Fathima, 2017; Phattarakul et al., 2012; Shaygany et al., 2012; Siddika et al., 2016; Yuan et al., 2012). Por tal motivo, el presente estudio analiza cuatro tratamientos con elementos menores y su influencia en la calidad molinera del arroz.

\section{MATERIALES Y MÉTODOS}

El experimento se realizó en la vereda Borriqueros, ubicada a $7^{\circ} 56^{\prime}$ de latitud norte y $72^{\circ} 36^{\prime}$ de longitud oeste, en el municipio del Zulia, departamento de Norte de Santander, Colombia. La zona presenta un clima semiárido cálido (según clasificación climática de Köppen: BSh), con altitud sobre el nivel del mar de 220 metros, temperatura media anual de $28^{\circ} \mathrm{C}$ y humedad relativa de $58 \%$. La investigación se realizó en un área de 2,5 ha, las cuales fueron divididas en cuatro piscinas cultivadas con arroz variedad Fedearroz 2000, estableciendo cinco bloques de $36 \mathrm{~m}^{2}$ para cada tratamiento.

\section{Preparación del lote para la siembra}

Se realizó la nivelación del lote mediante la utilización de pala láser. Antes de la siembra se implementó un pase de rastra para mejorar la aireación del suelo e incorporar los residuos del control de malezas. La prueba de germinación se realizó con 100 semillas tomadas al azar con tres replicas (Mercado et al., 2020; Salazar et al., 2020a; Salazar et al., 2020b; Salazar-Mercado et al., 2020). Todas estas semillas fueron previamente sumergidas en agua durante dos días para hidratarlas e inducir la germinación. La siembra se ejecutó con una densidad de $160 \mathrm{~kg} / \mathrm{ha}$ de forma manual al voleo.

\section{Plan de fertilización}

La fertilidad del lote se basó en los resultados arrojados por el plan integral de nutrición con tecnología satelital, conocido como PINSAT. En la primera fertilización (tabla 1) se aplicó fósforo y nitrógeno y se adicionaron elementos menores y una fracción de elementos secundarios. Así mismo, en el tratamiento T3 se realizó la primera aplicación del silicio edáfico. En la segunda fertilización se realizó la segunda aplicación de silicio edáfico en el T3. Igualmente, se realizó la aplicación de fósforo y nitrógeno como elementos esenciales, más la adición de magnesio y azufre. Para la tercera fertilización se adicionó la tercera fracción de nitrógeno y potasio más la última fracción de calcio y boro. En la cuarta fertilización se adicionó nitrógeno y potasio en partes iguales, con pequeñas fracciones de azufre y magnesio. Las aplicaciones foliares (tabla 2) se realizaron 45 y 55 días después del germinado (d. d. g.) en máximo macollamiento y tras la aparición del anillo verde del arroz.

El ensayo se realizó bajo un sistema convencional de siembra con lámina de agua de $5 \mathrm{~cm}$ durante 24 horas. Después de las 48 horas de sembrado se utilizó riego corrido (mojes) cada dos días hasta la primera fertilización. Posteriormente, se dejó una lámina de riego de 5 $\mathrm{cm}$ para el control de arvenses. A los 25 días de germinado se dejó constante una lámina de $10 \mathrm{~cm}$ y a partir de los 105 días no se llevó a cabo riego alguno. 
Tabla 1. Fertilización edáfica aplicada por tratamiento, lote Borriqueros, El Zulia, Norte de Santander

\begin{tabular}{|c|c|c|c|c|c|c|c|c|c|c|c|c|}
\hline \multirow[b]{2}{*}{ Tratamiento } & \multirow{2}{*}{$\begin{array}{c}\text { Numero de } \\
\text { abonadas }\end{array}$} & \multirow{2}{*}{$\begin{array}{c}\text { Dosis total } \\
\mathrm{kg} / \mathrm{ha} \\
\text { abonada }\end{array}$} & \multicolumn{10}{|c|}{ Producto kg/ha } \\
\hline & & & Abotain & DAP & $\mathrm{KCl}$ & Rafos & $\begin{array}{l}\text { Sulfato } \\
\text { Fe }\end{array}$ & $\begin{array}{l}\text { Cubo } \\
\text { ZInc }\end{array}$ & Sulfato K & Sulpomag & $\mathrm{Nica}+\mathrm{B}$ & $\begin{array}{c}\text { Llanero } \\
\text { ZeO }\end{array}$ \\
\hline \multirow{4}{*}{$\mathrm{T} 1$} & 1 & 110 & 31,5 & 78,5 & - & - & - & - & - & - & - & - \\
\hline & 2 & 192,5 & 157 & - & 31,5 & - & - & - & - & - & - & - \\
\hline & 3 & 173 & 63 & - & 110 & - & - & - & - & - & - & - \\
\hline & 4 & 110 & 31 & - & 78 & - & - & - & - & - & - & - \\
\hline \multirow{4}{*}{$\mathrm{T} 2$} & 1 & 181,5 & - & - & - & 150 & 10 & 10 & - & - & - & - \\
\hline & 2 & 175 & 100 & - & - & - & - & - & 25 & 50 & - & - \\
\hline & 3 & 225 & 100 & - & 75 & - & - & - & - & - & 50 & - \\
\hline & 4 & 125 & 50 & - & 50 & - & - & - & - & 25 & - & - \\
\hline \multirow{4}{*}{ T3 } & 1 & 170 & - & - & - & 150 & 10 & 10 & - & - & - & 25 \\
\hline & 2 & 175 & 100 & - & - & - & - & - & 25 & 50 & - & 25 \\
\hline & 3 & 225 & 100 & - & 75 & - & - & - & - & - & 50 & - \\
\hline & 4 & 125 & 50 & - & 50 & - & - & - & - & 25 & - & - \\
\hline \multirow{4}{*}{ T4 } & 1 & 170 & - & - & - & 150 & 10 & 10 & - & - & - & - \\
\hline & 2 & 175 & 100 & - & - & - & - & - & 25 & 50 & - & - \\
\hline & 3 & 225 & 100 & - & 75 & - & - & - & - & - & 50 & - \\
\hline & 4 & 125 & 50 & - & 50 & - & - & - & - & 25 & - & - \\
\hline \multirow{4}{*}{ T5 } & 1 & 119,3 & 31,5 & 78,5 & - & - & - & - & - & - & - & - \\
\hline & 2 & 188,5 & 157 & - & 31,5 & - & - & - & - & - & - & - \\
\hline & 3 & 199,4 & 63 & - & 110 & - & - & - & - & - & - & - \\
\hline & 4 & 88 & 31 & - & 78 & - & - & - & - & - & - & - \\
\hline
\end{tabular}

Abotain (N $46 \%$ ); DAP (N $18 \%$, P $46 \%$ ); KCL (Cl $46 \%$ ); Rafos (N $12 \%$, P $24 \%$, K $12 \%$, Mg $2 \%$, S $1 \%$, B 0,04 \%); Sulfato Fe (Fe $20 \%$, S $11 \%$ ); Cubozinc (N $5 \%$, Ca $2 \%$, Mg $6 \%$, S $9 \%$, B 2,5 \%, Cu 0,5 \%, Zn 16 \%); Sulfato K (K 50 \%, S 18 \%); Sulpomag (K 22 \%, Mg 18 \%, S 21,5 \%, Cl 2,5 \%); Nica + B (N 15 \%, P 4 \%, K 4 \%); Llanero Zeo (Si 78 \%).

Fuente: elaboración propia.

La investigación utilizó un diseño de bloques completos al azar para cinco tratamientos con cuatro repeticiones (cada uno) en un área de $36 \mathrm{~m}^{2}$, para un total de $180 \mathrm{~m}^{2}$ por tratamiento. Los factores a evaluar en los tratamientos son elementos menores aplicados al suelo (EMS), elementos menores aplicados al follaje (EMF) en combinación con silicio aplicado al suelo (SS), silicio aplicado al follaje (SF) y un adicional que fungirá como testigo (TT), donde se realizó fertilización convencional. Este estudio se realizó considerando cinco tratamientos:

\section{T1: fertilización tradicional con NPK.}

T2: fertilización tradicional NPK + aplicación edáfica de elementos menores ( $\mathrm{Ca}, \mathrm{Mg}, \mathrm{S}, \mathrm{Fe}, \mathrm{Mn}$, $\mathrm{Cu}, \mathrm{Zn}, \mathrm{Cl}, \mathrm{B}, \mathrm{Mo})$.
T3: fertilización tradicional NPK + aplicación edáfica de elementos menores $(\mathrm{Ca}, \mathrm{Mg}, \mathrm{S}, \mathrm{Fe}, \mathrm{Mn}$, $\mathrm{Cu}, \mathrm{Zn}, \mathrm{Cl}, \mathrm{B}, \mathrm{Mo})+$ aplicación edáfica de silicio.

T4: fertilización tradicional NPK + aplicación edáfica de elementos menores ( $\mathrm{Ca}, \mathrm{Mg}, \mathrm{S}, \mathrm{Fe}, \mathrm{Mn}$, $\mathrm{Cu}, \mathrm{Zn}, \mathrm{Cl}, \mathrm{B}, \mathrm{Mo}$ ) + aplicación foliar de silicio.

T5: fertilización tradicional NPK + aplicación foliar de elementos menores ( $\mathrm{Ca}, \mathrm{Mg}, \mathrm{S}, \mathrm{Fe}, \mathrm{Mn}, \mathrm{Cu}$, $\mathrm{Zn}, \mathrm{Cl}, \mathrm{B}, \mathrm{Mo})+$ aplicación foliar de silicio.

Los datos se corrieron en el sistema de análisis estadístico SAS, versión 9.3, donde se realizó análisis de varianza (ANOVA) y pruebas de Tukey. Los tratamientos 1,2 y 3 no aparecen en la tabla de dosificación puesto que a estos no se les aplicó fertilización foliar. 
Tabla 2. Fertilización foliar aplicada por tratamientos

\begin{tabular}{|c|c|c|c|c|}
\hline \multirow{2}{*}{ Tratamiento } & \multirow{2}{*}{$\mathrm{N} .^{\circ}$ de fertilizaciones } & \multirow{2}{*}{$\begin{array}{l}\text { Dosis total (I) por ha } \\
\text { fertilizada }\end{array}$} & \multicolumn{2}{|c|}{ Producto $\mathrm{I} /$ tratamiento } \\
\hline & & & Nitrosil-K* & Novaplant** \\
\hline \multirow{4}{*}{ T4 } & 1 & - & - & - \\
\hline & 2 & - & - & - \\
\hline & 3 & 0,5 & 0,5 & - \\
\hline & 4 & 0,5 & 0,5 & - \\
\hline \multirow{4}{*}{ T5 } & 1 & - & - & - \\
\hline & 2 & - & - & - \\
\hline & 3 & 1 & 0,5 & 0,5 \\
\hline & 4 & 1 & 0,5 & 0,5 \\
\hline
\end{tabular}

*Nitrosil-K: suministra dosis de silicio al tratamiento.

**Novaplant: suministra elementos menores al tratamiento.

Fuente: elaboración propia.

\section{Calidad molinera del arroz}

Para evaluar el componente de molinería (prueba de trilla) se utilizaron $\mathbf{5 0 0}$ gramos de granos por bloque. Las variables de producción fueron: densidad de plantas (plantas $/ \mathrm{m}^{2}$ ), macollamiento (macollas $/ \mathrm{m}^{2}$ ) y número de panículas por área $\left(\mathrm{m}^{2}\right)$. Dichas variables se determinaron arrojando al azar dentro de cada tratamiento un marco de aforo de un metro cuadrado $\left(1 \mathrm{~m}^{2}\right)$, el cual es elaborado con cuatro varas de 1 metro de largo unidas por sus extremos, formando exactamente un cuadro. Se tomaron 6 panículas al azar por réplica, 24 panículas por tratamientos. Se seleccionó el número de granos totales (NGT) de las panículas para determinar el número de espiguillas. Posteriormente, por panícula se contó el total de granos y estos se dividieron en número de granos llenos (NGLI) y número de granos vanos (NGV) para así determinar el porcentaje de vaneamiento (\%Vano), el cual fue calculado a partir de la siguiente fórmula:

$$
\text { Vaneamiento }=\left(\frac{\text { Número de granos totales }}{\text { Número de granos vanos }}\right) \times 100
$$

\section{Rendimiento del arroz}

La muestra representativa de cada bloque estuvo compuesta por seis plantas de cada tratamiento para los componentes de rendimiento, teniendo en cuenta el efecto de borde. Para determinar el rendimiento $(\mathrm{kg} / \mathrm{ha})$ se cosechó y se pesó cada tratamiento por separado. La evaluación de los parámetros de calidad molinera involucró el uso de los equipos de laboratorio ubicados en las instalaciones del Molino Oro, propiedad de COAGRONORTE. El parámetro a tener en cuenta dentro de la calidad molinera es el rendimiento de pilada (rendimiento/pilada). Para hallar esta variable es necesario realizar el pesaje de $100 \mathrm{gr}$ de arroz paddy con una humedad entre 11,5 y $12,5 \%$. Posteriormente, esta muestra es pasada por la descascaradora, para luego ser pesada, pulida y pesada una vez más. Por último, la muestra es analizada en scanner tipo SATAKE RSQI, del cual se obtiene el porcentaje de masa blanca (\%MB), grano entero (\%GE), grano cristal (\%CRIS), harina (\%HAR), cascarilla (\%CAS) y grano yesado (\%GY) del $100 \%$ de la muestra de arroz paddy, esta vez seco. 
Tabla 3. Valor F del análisis de varianza para cada variable evaluada

\begin{tabular}{|c|c|c|c|c|c|c|c|c|c|}
\hline Variable & Plantas $/ \mathrm{m}^{2}$ & Macollas $/ \mathrm{m}^{2}$ & Pani/m & NEspi & NGV & NGLL & NGT & $\%$ Vano & $\mathrm{kg} / \mathrm{ha}$ \\
\hline GL & 4 & 4 & 4 & 4 & 4 & 4 & 4 & 4 & 4 \\
\hline F Value & $46,27^{*}$ & 59,48 & 8,53 & 2,76 & 19,88 & 7,88 & 15,44 & 15,24 & $37,19 *$ \\
\hline Variable & $\% \mathrm{MB}$ & $\%$ GE & $\%$ CRIS & $\%$ HAR & $\%$ CAS & $\%$ GY & & & \\
\hline GL & 4 & 4 & 4 & 4 & 4 & 4 & & & \\
\hline F Value & $70,57^{*}$ & $446,9 *$ & $176,93^{*}$ & $79 *$ & 7,89 & $82,08^{*}$ & & & \\
\hline
\end{tabular}

* Probabilidad < 0.0001; GL: grados de libertad.

Fuente: elaboración propia.

\section{RESULTADOS Y DISCUSIÓN}

El análisis de varianza reportó diferencias altamente significativas entre los tratamientos evaluados $(\mathrm{p}<0,0001)$ para las siguientes variables: plantas $/ \mathrm{m}^{2}$, $\mathrm{kg} / \mathrm{ha}, \% \mathrm{MB}, \% \mathrm{GE}, \% \mathrm{CRIS}$, \%HAR y \%GY (tabla 3).

En la investigación se evaluaron cinco fertilizaciones diferentes enfocadas a la utilización de elementos menores con respecto al plan nutricional normalmente utilizado por los agricultores del sector, llevando a cabo un total de cuatro réplicas del tratamiento en la zona. Se observó que la variable densidad de plantas/ $\mathrm{m}^{2}$ presentó diferencias significativas $(\mathrm{p}<0,0001)$ entre los tratamientos (figura $1 \mathrm{~A}$ ), siendo $\mathrm{T} 3$ el que presentó el mayor valor $\left(396,21\right.$ plantas $\left./ \mathrm{m}^{2}\right)$, seguido de T5 y T4 (388,16 y 386,81 plantas $/ \mathrm{m}^{2}$, respectivamente). En contraste T1 $\left(336,34\right.$ plantas $\left./ \mathrm{m}^{2}\right)$ y T2 $(313,49$ plantas $/ \mathrm{m}^{2}$ ) alcanzaron los valores más bajos. Según lo reportado por Ashrafi et al. (2014), estos valores manifiestan resultados no significativos debido a que el distanciamiento entre plantas puede verse afectado por la baja cantidad de micronutrientes y el exceso de agua aplicada en los lotes. Por su parte, Ramírez et al. (2017) encontraron que al reducir el espaciamiento disminuye el peso de las panículas.

La variable macollamiento $/ \mathrm{m}^{2}$ presentó diferencias significativas $(p 0,0002)$ entre los tratamientos (figura 1B), siendo T4 el de mayor valor (1.221,18 macollas $\left./ \mathrm{m}^{2}\right)$, mientras que T1 reportó el valor más bajo $\left(821,26\right.$ macollas $\left./ \mathrm{m}^{2}\right)$. Estos resultados concuerdan con lo reportado por Radhika et al. (2013) y Siddika et al. (2016), quienes obtuvieron mayor número de macollas productivas en cultivos de arroz tras la aplicación de micronutrientes y silicio dentro de los planes de fertilización.
Así mismo, la variable panículas $/ \mathrm{m}^{2}$ mostró diferencias significativas (p 0,0119) entre tratamientos (figura $1 \mathrm{C})$, siendo T2 $\left(709,09\right.$ panículas $\left./ \mathrm{m}^{2}\right)$ y T3 $\left(705,29\right.$ panículas $\left./ \mathrm{m}^{2}\right)$ aquellos con los valores más altos. Por otro lado, T5 reportó el menor valor $(638,66$ panículas $/ \mathrm{m}^{2}$ ). Estos hallazgos fueron mejores a los expuestos por Álvarez et al. (2008), Hansch y Mendel (2009), Kumar (2014) y Kumar et al. (2015), quienes observaron que la aplicación adecuada de nutrientes es indispensable para obtener cosechas de mayores rendimientos.

La variable número de espiguillas/panícula no presentó diferencias significativas ( $p 0,0773)$ entre los tratamientos (figura 1D), siendo T5 (10 espiguillas/ panícula) el de menor valor y T4 (12,24 espiguillas/ panícula) el de mayor valor. Estos resultados contrastan con lo reportado por estudios como los de Yruela (2005), Yamasaki et al. (2008), Ahmad et al. (2009), Roholla et al. (2011), Hafeez et al. (2013) y Rout y Sahoo (2015), los cuales manifiestan que la aplicación de microelementos no tiene incidencia en el número de espiguillas por panícula.

De otro lado, la variable número de granos llenos (NGLL) presentó diferencias significativas (p 0,003) entre tratamientos (figura $1 \mathrm{E}$ ), en donde $\mathrm{T} 2$ presentó el valor más alto (126,32 granos llenos/panícula) y T1 el más bajo (99,53 granos llenos/panícula). Estos hallazgos coinciden con lo reportado por Álvarez et al. (2008), Jin et al. (2008), Phattarakul et al. (2012) y Yuan et al. (2012), quienes expresan que siempre que se aplica silicio aumenta en calidad y peso el número de granos llenos.

La variable número de granos vanos (NGV) también presentó diferencias significativas ( $p$ 0.0003) entre tratamientos (figura 1F), siendo T1 aquel con el mayor valor (22,28 granos vanos/panícula) y T2 el de menor 
valor (10,43 granos vanos/panícula). Estos resultados son similares a los de Álvarez et al. (2008), Jin et al. (2008), Phattarakul et al. (2012) y Yuan et al. (2012), puesto que, como señalan estos autores, al aplicar elementos menores y silicio vía edáfica aumenta el número de granos llenos.

En la misma línea, la variable número de granos totales por panícula (NGT) presentó diferencias significativas ( $p$ 0.0002) entre tratamientos (figura $1 \mathrm{G}$ ), donde T4 $(138,77)$ presentó el mayor número de granos totales por panícula, mientras que T1 $(121,84)$ y T5 $(117,53)$ reportaron los valores más bajos. Los resultados para esta variable concuerdan con lo expresado por Meena y Fathima (2017) y Saha et al. (2017), quienes manifiestan que la aplicación de elementos al suelo es importante para la obtención de un mayor número de granos por panícula, aunque esta debe ser complementada con aplicaciones vía foliar.

En cuanto a la variable porcentaje de vaneamiento (\%Vano), también se observaron diferencias significativas ( 0 ,0003) entre los tratamientos (figura $1 \mathrm{H}$ ). Así, T1 presentó el mayor porcentaje de vaneamiento por panícula $(18,48 \%)$ y T2 el menor $(7,74 \%)$. Estos resultados se asemejan a lo presentado por Meena y Fathima (2017), Saha et al. (2017) y Balindong et al. (2018), quienes expresan que la aplicación de fertilizantes al suelo no es suficiente para garantizar el llenado de granos y frutos.

Adicionalmente, se observó que la variable rendimiento $(\mathrm{kg} / \mathrm{ha})$ presentó diferencias significativas $(p<0,0001$ ) entre tratamientos (figura 11), siendo T2 el mejor tratamiento en producción $(7.689 \mathrm{~kg} / \mathrm{ha})$ y T1 el de menor producción $(6.886 \mathrm{~kg} / \mathrm{ha})$. En el estudio realizado por Álvarez et al. (2008), la variable de rendimiento de arroz paddy en la localidad de lbagué presentó rendimientos de $8.558 \mathrm{~kg} / \mathrm{ha}$, mientras que en la localidad de El Guamo esta cifra alcanzó 6.107 kg/ ha. Por ende, se puede concluir que el comportamiento de esta variable en la zona de Borriqueros fue aceptable, en comparación con las otras localidades mencionadas. Los resultados obtenidos en esta investigación son similares a los de Shaygany et al. (2012), quienes obtuvieron un rendimiento de grano de hasta 6,17 t/ha para fertilización con micronutrientes, en contraste con 4,51 t/ha en el tratamiento control. Del mismo modo, Alamdari y Mobasser (2014) reportaron rendimientos promedios de hasta $4,3 \mathrm{t} / \mathrm{ha}$ con la aplicación de microelementos, en comparación con $2,8 \mathrm{t} / \mathrm{ha}$ en el tratamiento testigo.

Figura 1. Análisis de las medias de cada variable

\section{A. Plantas $/ \mathrm{m}^{2}$}

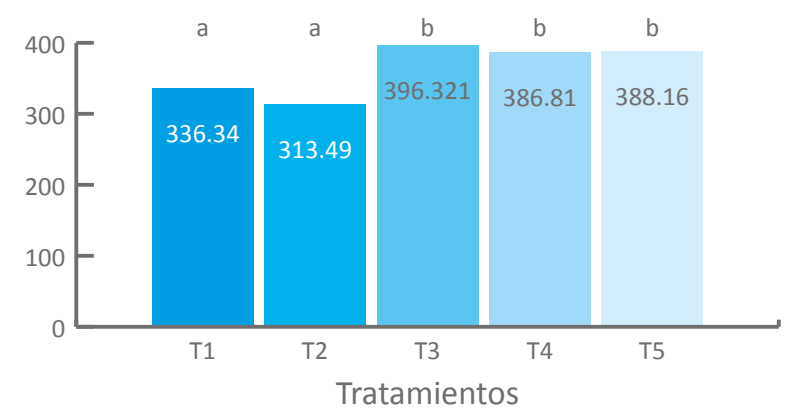

\section{B. Macollamiento/ $\mathrm{m}^{2}$}

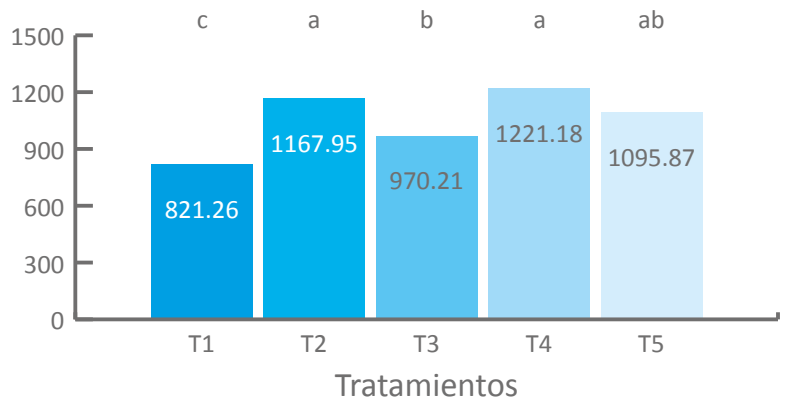

\section{Panículas $/ \mathrm{m}^{2}$}

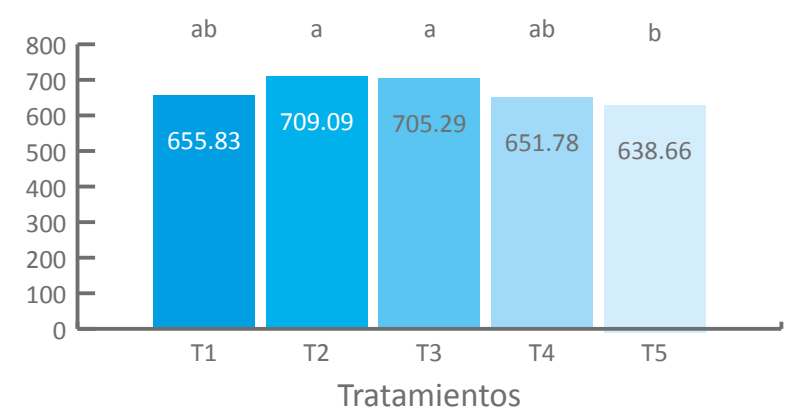

D. Números de espiguillas/panícula

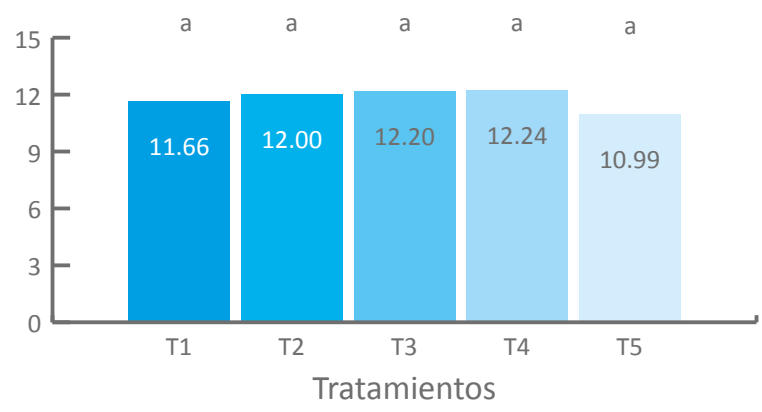


E. Números de granos llenos/panícula

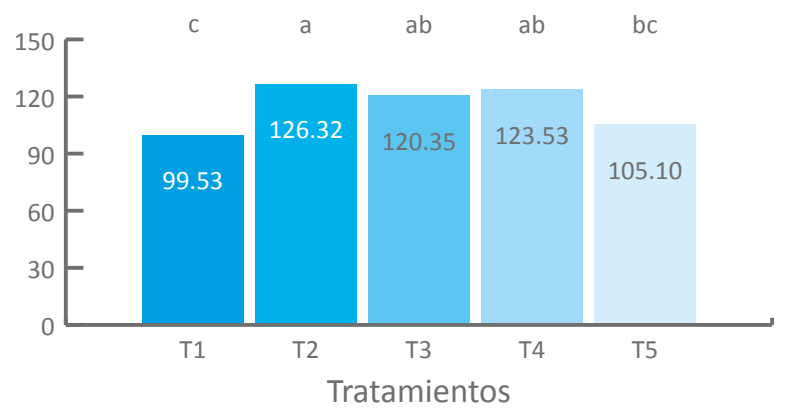

F. Número de granos vanos/panícula

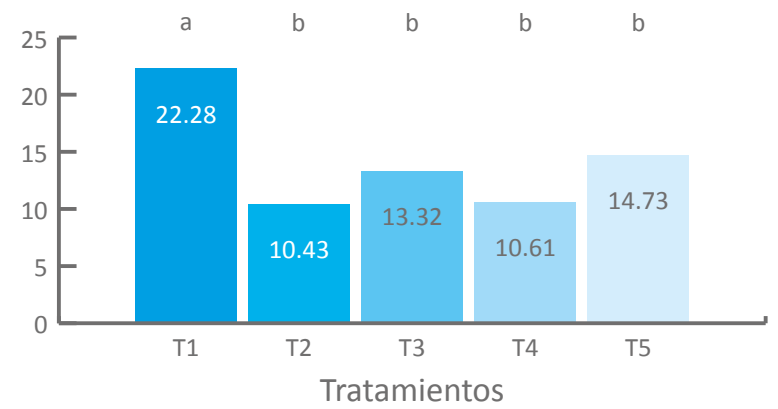

G. Número de granos totales/panícula

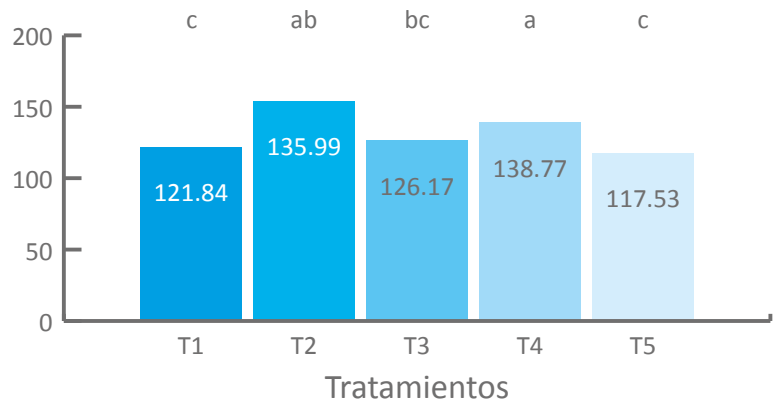

H. Vaneamiento

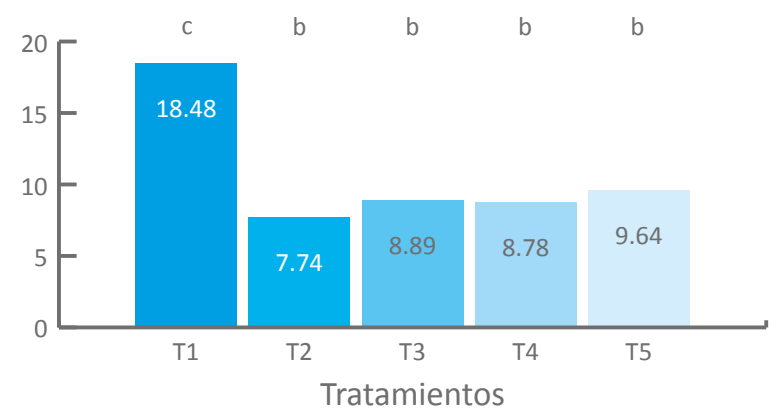

I. $\mathrm{Kg} / \mathrm{ha}$

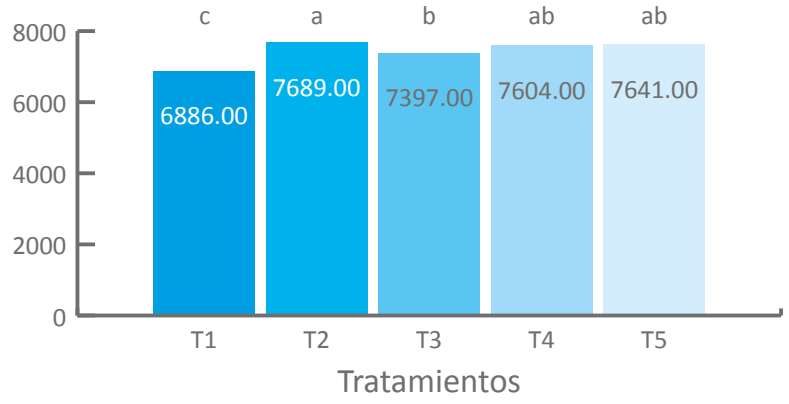

A. Densidad de plantas $/ \mathrm{m}^{2}$; B. Macollamiento $/ \mathrm{m}^{2} ;$ C. Panículas $/ \mathrm{m}^{2} ; \mathrm{D}$. Número de espiguillas/panícula; E. Número de granos llenos/panícula; F. Número de granos vanos/panícula; G. Número de granos totales/panícula; H. Porcentaje de vaneamiento; I. Porcentaje de rendimiento ( $\mathrm{kg} / \mathrm{ha})$.

Fuente: elaboración propia.

Para las variables de producción se tuvo en cuenta los requerimientos arrojados por el análisis PINSAT, obteniendo que el tratamiento con mejor desempeño fue T2 (aplicación de NPK + elementos menores edáficos), cuya dosificación mostró datos importantes para la variable de panículas $/ \mathrm{m}^{2}$, número de granos llenos/ panícula y rendimiento $\mathrm{kg} / \mathrm{ha}$. De otro lado, la utilización de un plan nutricional adecuado, como T4 (aplicación de NPK + elementos menores edáficos + silicio foliar), evidenció que la variable de macollamiento/ $\mathrm{m}^{2}$ y número de granos totales incrementa la producción de arroz. Estos datos coinciden con los obtenidos por Rodríguez-Araujo et al. (2010), Shayganya et al. (2012), quienes aconsejan hacer aplicaciones de elementos mayores y menores complementadas con aplicaciones foliares, además del uso de silicio como reconstituyente de suelos.

\section{Variables de molinería}

La variable porcentaje de masa blanca reportó diferencias significativas $(p<0,0001)$ entre tratamientos (figura $2 \mathrm{~A}$ ), siendo T5 el que obtuvo el mayor porcentaje $(70,68 \%)$ y T1 el menor $(66,25 \%)$. Estos datos concuerdan con lo presentado por Mejía y Menjivar (2010), Domínguez y Muñoz (2012) y Livore (2013), quienes manifiestan que la realización de evaluaciones de calidad molinera es necesaria para determinar la calidad de las especies de arroz sembradas alrededor del mundo. 
Igualmente, la variable porcentaje de granos enteros registró diferencias significativas $(p<0,0001)$ entre tratamientos (figura 2B), siendo T5 el de mayor porcentaje $(59,65 \%)$ y T1 aquel con el menor resultado $(40,96 \%)$. Estos hallazgos son similares a los reportados por Miranda y Ruíz (2015), quienes expresan que los planes de fertilización que involucran la aplicación de elementos mayores y menores, complementados con la adición de productos foliares, garantizan granos enteros que son bien comercializados.

La variable porcentaje de cristal (grano partido) también registró diferencias significativas $(p<0,0001)$ entre tratamientos (figura $2 \mathrm{C}$ ), donde $\mathrm{T} 1$ obtuvo el mayor porcentaje de granos partidos $(25,24 \%)$ y T3 el menor porcentaje (11,09\%). Resultados similares a los de Martínez et al. (2010) y Jo y Todorov (2019), quienes manifiestan que el uso de fertilizantes compuestos a base de NPK, complementados con micronutrientes, incrementa de manera notable los componentes del rendimiento y el rendimiento general del cultivo de arroz.

Igual que con las variables anteriores, el porcentaje de harina reportó diferencias significativas $(p<0,0001)$ entre tratamientos (figura 2D), siendo T1 el de mayor porcentaje $(12,82 \%)$ y T5 el de la cifra más baja $(9,32$ $\%)$. Estos hallazgos coinciden con los resultados de Larios et al. (2010), quienes expresan que la fertilización con micronutrientes no solo mejora notablemente el rendimiento del cultivo, sino que además puede incrementar la calidad nutricional del grano, lo cual resulta importante y atractivo para los consumidores desde la perspectiva de la salud humana, puesto que adquirir un producto biofortificado es clave para contrarrestar problemas de desnutrición.

En la variable porcentaje de cáscara se observaron diferencias significativas $(p 0,0023)$ entre tratamientos (figura 2E), donde $\mathrm{T} 1$ registró el mayor valor $(20,85$ $\%)$ y $\mathrm{T} 5$ el menor porcentaje $(19,95 \%)$. Estos resultados son similares a los observados por Siebenmorgen (2013), autor que establece que la fertilización convencional a base de NPK es imprescindible para mejorar la calidad molinera del grano de arroz.

Para la variable porcentaje de yesado también se observaron diferencias significativas $(p<0.0001)$ entre tratamientos (figura $2 \mathrm{~F}$ ), siendo $\mathrm{T} 2$ aquel con el mayor porcentaje $(22,18 \%)$. Por su parte, el porcentaje de esta variable en los demás tratamientos fue mínimo, sin superar $1 \%$ de yesado. Esto es similar a lo presentado por Guillermo et al. (2012) y Zhang et al. (2017), quienes manifestaron que la inclusión de micronutrientes en los planes de fertilización convencional basados en NPK puede incrementar notablemente el rendimiento y la calidad nutraceútica del grano de arroz.

Figura 2. Análisis de las medias de cada variable de molinería

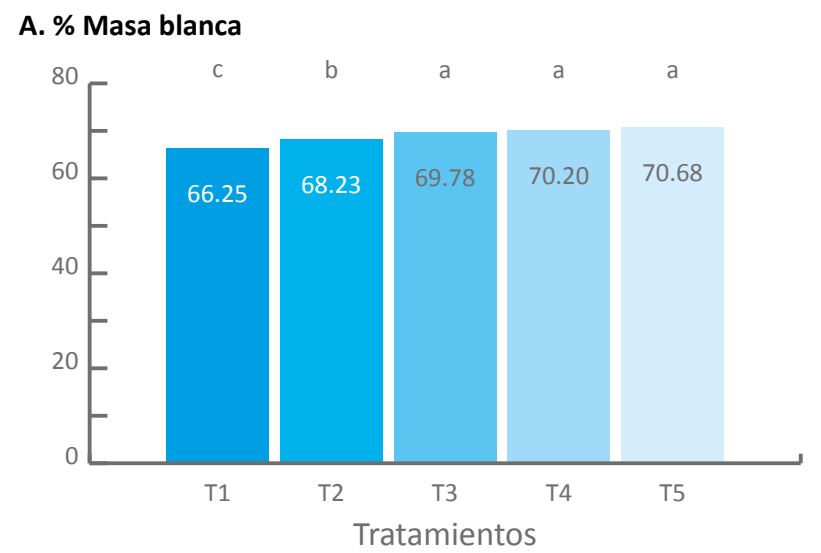

B. \% Grano entero

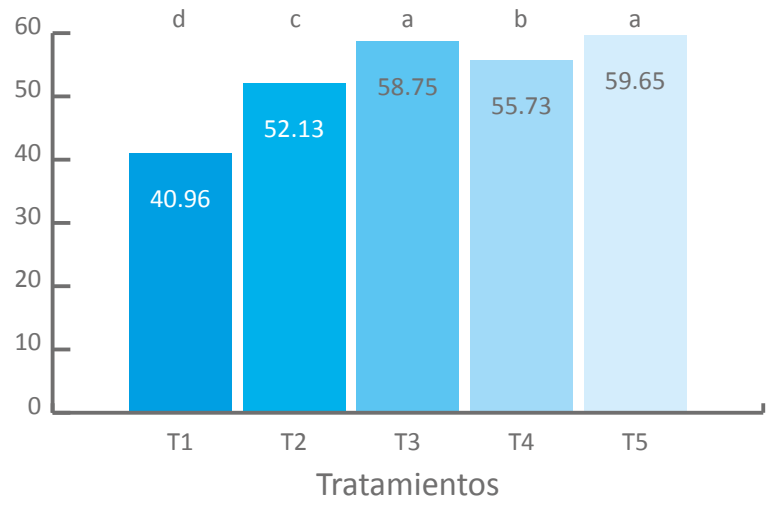

C. \% Cristal





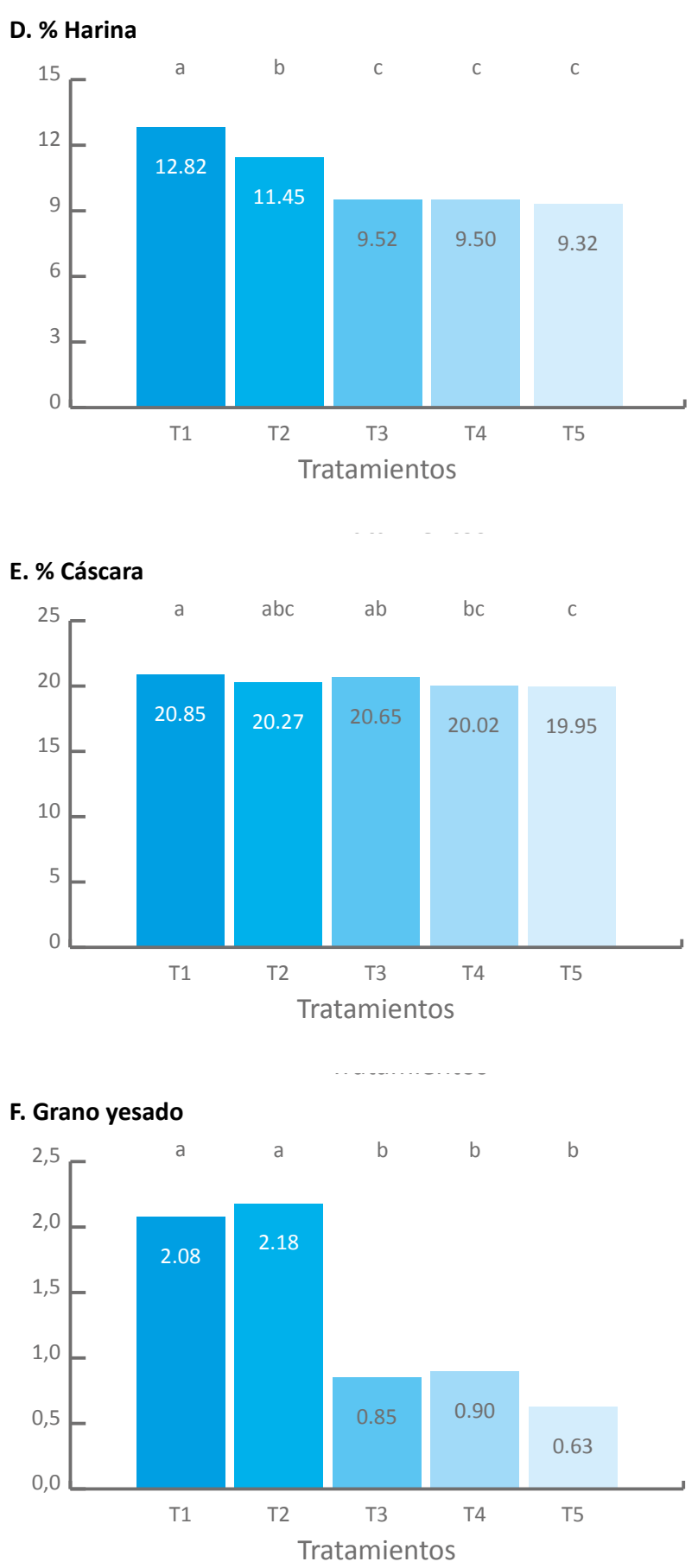

A. Porcentaje de masa blanca; B. Porcentaje de grano entero; C. Porcentaje de cristal; D. Porcentaje de harina; E. Porcentaje de cáscara; F. Porcentaje de grano yesado.

Fuente: elaboración propia.
Para las variables de calidad molinera el tratamiento con los mejores resultados fue T5 (aplicación de NPK + elementos menores foliares + silicio foliar), el cual demostró buenos efectos sobre las variables porcentaje de masa blanca y porcentaje de grano entero, así como la menor cantidad de granos yesados, cristal y harina. Estos datos que concuerdan con lo expuesto por Mejía y Menjivar (2010), Abubakar et al. (2018), quienes expresan que la utilización de fertilización convencional basada en NPK y la adición de elementos menores y silicio mejora la calidad molinera de la variedad Fedearroz 2000.

\section{CONCLUSIONES}

Se observó que el tratamiento con mejor comportamiento en cuanto a las variables de producción y rendimiento fue T2 (fertilización NPK + fertilización de elementos menores edáficos), debido a que este mostró un alto grado de aceptación para las variables de panículas $/ \mathrm{m}^{2}$, número de granos llenos/panícula y rendimiento $(\mathrm{kg} / \mathrm{ha})$, en comparación con el testigo (T1), que corresponde al esquema de fertilización más empleado en la zona de Borriqueros.

Se evidenció que a nivel de calidad molinera el tratamiento con mejor comportamiento fue T5 (fertilización NPK + fertilización con elementos menores foliar + fertilización foliar con silicio), el cual permite mejorar los porcentajes de masa blanca y granos enteros, así como obtener el menor porcentaje de granos yesados. Además, la utilización de elementos menores (en especial el silicio) permite fortalecer el sistema inmune de la planta, contribuyendo a combatir agentes bióticos causantes del vaneamiento y comprobando así la importancia de aplicar elementos menores al suelo, los cuales producen una respuesta positiva sobre la calidad molinera del arroz.

La aplicación de fertilizantes NPK + fertilización con elementos menores foliar + fertilización foliar con silicio mejora las condiciones de producción y rendimiento del arroz, lo cual se ve traducido en mejores ingresos económicos para el productor y la entrega de un producto de mejor calidad para el consumo humano. 


\section{REFERENCIAS}

Abubakar, B., Yakasai, H. M., Zawawi, N., \& Ismail, M. (2018). Compositional analyses of white, brown and germinated forms of popular Malaysian rice to offer insight into the growing diet-related diseases. Journal of Food and Drug Analysis, 26(2),706-715. https://doi.org/10.1016/j. jfda.2017.06.010

Ahmad, W., Niaz, N., Kanwal, S., \& Khalid, M. (2009). Role of boron in plant growth: A review. J Agric Res., 47(3), 329-338.

Alamdari, M., \& Mobasser, H. (2014). The effect of macro and micro-nutrient fertilizers on yield and yield attributes of rice in a calcareous soil. American Journal of Experimental Agriculture, 4(12), 1604-1615. https://doi.org/10.9734/ ajea/2014/3536

Alshaal, T., \& El-Ramady, H. (2017). Foliar Application: from Plant Nutrition to Biofortification. The Environment, Biodiversity \& Soil Security, 1, 71-83. https://doi.org/10.21608/jenvbs.2017.1089.1006

Álvarez, J., Daza, M., \& Mendoza, C. (2008). Aplicación de un fertilizante enriquecido con silicio y materia orgánica en arroz (Oryza sativa L) cultivado en Ibagué y el Guamo (Tolima, Colombia). Revista Facultad Nacional Agronomía Medellín, 61(2), 4605-4617.

Ashrafi, E., Pirdashti, H., \& Niknejhad, Y. (2014). Effect of iron, zinc and silicon application on quantitative parameters of rice (Oryza Sativa L. CV. Tarom Mahalli). Intl J Farm \& Alli Sci., 3(5), 529-533.

Balindong, J. L., Ward, R. M., Liu, L., Rose, T. J., Pallas, L. A., Ovenden, B. W., Snell, P. J., \& Waters, D. L. (2018). Rice grain protein composition influences instrumental measures of rice cooking and eating quality. Rev. Cereal, 79(18), 35-42. https://doi. org/10.1016/j.jcs.2017.09.008

Barona, E. (2010). Importancia de la semilla de arroz. Arroz, 58(489), 15-21.
Bashir, K., Takahashi, R., Nakanishi, H., \& Nishizawa, N. (2013). The road to micronutrient biofortification of rice: Progress and prospects. Frontiers in Plant Science, 4(15), 1-7. https://doi. org/10.3389/fpls.2013.00015

Botta, G. F., Tolón-Becerra, A., Lastra-Bravo, X., Hidalgo, R., Rivero, D., \& Agnes, D. (2015). Alternatives for handling rice (Oryza sativa L.) straw to favor its decomposition in direct sowing systems and their incidence on soil compaction. Geoderma, 239-240, 213-222. https://doi. org/10.1016/j.geoderma.2014.10.021

Bouis, H., \& Saltzman, A. (2017). Improving nutrition through biofortification: A review of evidence from HarvestPlus, 2003 through 2016. Global Food Security, 12, 49-58. https://doi. org/10.1016/j.gfs.2017.01.009

Cakmak, I., \& Kutman, U. (2017). Agronomic biofortification of cereals with zinc: A review. European Journal of Soil Science, 69(1), 172-180. https://doi.org/10.1111/ejss.12437

Das, J., Salam, R., Kumar, R., \& Bhutta, Z. (2013). Micronutrient fortification of food and its impact on woman and child health: A systematic review. Systematic Reviews, 2, 67. https://doi. org/10.1186/2046-4053-2-67

Domínguez, A., \& Muñoz, O. (2012). Evaluación de la calidad molinera y dimensiones de los granos de dos variedades de arroz y sus varietales de arroz maleza. Rev. Agronomía Tropical Argentina, 2(12), 5-6.

Dutta, S., Das, S., Pale, G., langrai, B., Aochen, C., Rai, M., \& Pattanayak, A. (2016). Current status and future prospects of research on genetically modified rice: A review. Agricultural Reviews, 37(1), 10-18. https://doi.org/10.18805/ ar.v37i1.9259

Fairhurst, T. H., \& Witt, C. (2002). Guía práctica para el manejo de nutrientes. Postash \& Phosphate Institute (PPI), Potash \& Phosphate Institute of Canada (PPIC), \& International Rice Research Institute (IRRI). 
Food and Agriculture Organization of the United Nations [FAO]. (2009). Global agriculture towards 2050: High Level Expert Forum - How to Feed the World in 2050. FAO http://www.fao.org/ fileadmin/templates/wsfs/docs/Issues_papers/ HLEF2050_Global_Agriculture.pdf

Food and Agriculture Organization of the United Nations [FAO]. (2014). A regional rice strategy for sustainable food security in Asia and the Pacific. FAO.

Gnanamanickam, S. (2009). Rice and its importance to human life. Prog Biol Con., 8, 1-11. https://doi. org/10.1007/978-90-481-2465-7_1

Guillermo, S., Acosta, C., Cleva, M., \& Gonzáles, A. (2012). Sistema de control de calidad de granos de arroz pulidos empleando visión por computadora. XIV Workshop de Investigadores en Ciencias de la Computación. Universidad Nacional de Misiones. http://sedici.unlp. edu.ar/bitstream/handle/10915/18840/ Sistema+de+control+de+calidad+de+granos+de +arroz+pulidos. pdf;jsessionid=69E2259FFC4A7D6F3899E8F1ED4D7C7E? sequence $=1$

Hafeez, B., Khanif, Y., \& Saleem, M. (2013). Role of zinc in plant nutrition. A review. American Journal of Experimental Agriculture, 3(2), 374-391. https://doi.org/10.9734/AJEA/2013/2746

Hansch, R., \& Mendel, R. (2009). Physiological functions of mineral micronutrients ( $\mathrm{Cu}, \mathrm{Zn}$, $\mathrm{Mn}, \mathrm{Fe}, \mathrm{Ni}, \mathrm{Mo}, \mathrm{B}, \mathrm{Cl})$. Current Opinion in Plant Biology, 12, 259-266. https://doi.org/10.1016/j. pbi.2009.05.006

Hernández-Rodríguez, A., Rives-Rodríguez, N., Acebo-Guerrero, Y., Diaz-de-la-Osa, A., Heydrich-Pérez, M., \& Divan-Baldani, L. (2014). Potencialidades de las bacterias diazotróficas asociativas en la promoción del crecimiento vegetal y el control de Pyricularia oryzae (Sacc.) en el cultivo del arroz (Oryza sativa L.) Revista de Protección Vegetal, 29(1), 1-10.

International Food Policy Research Institute [IFPRI]. (2014). Global nutrition report 2014: Actions and accountability to accelerate the world's progress on nutrition. IFPRI. https://doi. org/10.2499/9780896295803
Jin, Z., Minyan, W., Lianghuan, W., Jiangguo, W., \& Chunhai, S. (2008). Impacts of combination of foliar iron and boron application on iron biofortification and nutritional quality of rice grain. Journal of Plant Nutrition, 31, 1599-1611. https://doi.org/10.1080/01904160802244803

Jo, G., \& Todorov, T. I. (2019). Distribution of nutrient and toxic elements in brown and polished rice. Rev Food Chemistry., 289(2), 360-368. https://doi. org/10.1016/j.foodchem.2019.03.040

Kato, Y., \& Katsura, K. (2014). Rice adaptation to aerobic soils: Physiological considerations and implications for agronomy. Plant Production Sc., 17(1), 1-12. https://doi.org/10.1626/pps.17.1

Kumar, S. (2014). Role of micronutrient in rice cultivation and management strategy in organic agriculture. A reappraisal. Agricultural Sciences, 5, 765-769. https://doi.org/10.4236/as.2014.59080

Kumar, D., Singh, Sh., Singh, Sw., Mishra, S., Chauhan, D., \& Dubey, N. (2015). Micronutrients and their diverse role in agricultural crops: Advances and future prospective. Acta Physiol Plant., 37, 139. https://doi.org/10.1007/s11738-015-1870-3

Larios, A., Porcayo, J., \& Poggi, H. (2010). Obtención de una harina de pulido de arroz desengrasada con bajo contenido de fibra neutro detergente. Rev. Food Chemistry Colombia, 1(22), 3-5.

Livore, A. (2013). Calidad industrial y culinaria del arroz. IDIA XXI, 6, 190-194

Martínez, E., Pinciroli, M., \& Vidal, V. (2010). Proteínas de arroz propiedades estructurales y funcionales. Rev Argentina, 2(2),67-69.

Meena, N., \& Fathima, P. (2017). Nutrient uptake of rice as influenced by agronomic biofortification of $\mathrm{Zn}$ and Fe under methods of rice cultivation. Int J Pure App Biosci., 5(5), 456-459. https://doi. org/10.18782/2320-7051.3027

Mejía, M. S., \& Menjivar, J. C. (2010). Nutrición mineral de arroz. En V. M. Degiovanni-Beltramo, C. P. Martínez-Racines, \& F. Motta (eds.). Producción eco-eficiente del arroz en América Latina. Centro Internacional de Agricultura Tropical (CIAT). 
Mercado, S., Caleño, J., \& Rozo, L. (2020). Improvement of the methodology of the tetrazolium test using different pretreatments in seeds of the genus Epidendrum (Orchidaceae). Journal of Seed Science, 42, e202042013. https://doi. org/10.1590/2317-1545v42231028

Miranda, E., \& Ruíz, R. (2015). Análisis proximal de granos de arroz, frijol, maíz y café comercializados en el mercado. Revista Roberto Huembes de Managua, 6(18), 2-9.

Mohanty, S. (2013). Trends in global rice consumption. Rice Today, 12(1), 44-45.

Muthayya, S., Sugimoto, J., Montgomery, S., \& Maberly, G. (2014). An overview of global rice production, supply, trade, and consumption. Ann N. Y. Acad Sci, 1324, 7-14. https://doi. org/10.1111/nyas. 12540

Phattarakul, N., Rerkasem, B., Li, L., Hu, L., Zou, C., Ram, H., Sohu, B., Kang, B., Surek, H., Kalayci, M., Yazici, A., Zhang, F., \& Cakmak, I. (2012). Biofortification of rice grain with zinc through zinc fertilization in different countries. Plant Soil, 361, 131-141. https://doi.org/10.1007/ s11104-012-1211-x

Radhika, K., Hemalatha, S., Maragatham, S., \& Praveena, S. (2013). Effect of foliar application of micronutrients on the yield components of rice and soil available micronutrients status. Asian Journal of Soil Science, 8(2), 419-421.

Ramírez, E., Caraballo, R., \& Medina, V. (2017). Efecto de la distancia entre plantas y la época de siembra en las características morfológicas de la panícula de la planta de arroz (O. sativa L.). Instituto de Investigaciones del Arroz.

Rives, N., Acebo, Y., \& Hernández, A. (2007). Bacterias promotoras del crecimiento vegetal en el cultivo del arroz (Oryza sativa L.). Perspectivas de su uso en Cuba. Cultivos Tropicales, 28(2), 29-38.
Rodríguez-Araujo, E. A., Bolaños-Benavides, M. M., \& Menjivar-Flores, J. C. (2010). Efecto de la fertilización en la nutrición y rendimiento de ají (Capsicum spp.) en el Valle del Cauca, Colombia. Acta Agronómica, 59(1), 5564. https://www.redalyc.org/articulo. oa?id=1699/169916223005

Roholla, S., Shahsavari, M., \& Rezaei, M. (2011). A general overview on manganese (Mn) importance for crops production. Australian Journal of Basic and Applied Sciences, 5(9), 1799-1803.

Rout, G., \& Sahoo, S. (2015). Role of iron in plant growth and metabolism. Reviews in Agricultural Science, 3, 1-24. https://doi.org/10.7831/ras.3.1

Saha, S., Chakraborty, M., Padhan, D., Saha, B., Murmu, S., Batabyal, K., Seth, A., Hazra, G., Mandal, B., \& Bell, R. (2017). Agronomic biofortification of zinc in rice: Influence of cultivars and zinc application methods on grain yield and zinc bioavailability. Field Crops Research, 210, 52-60. https://doi.org/10.1016/j. fcr.2017.05.023

Salazar, S. A., Botello, H., \& Quintero, J. (2020a). Optimización de la prueba de tetrazolio para evaluar la viabilidad en semillas de Solanum lycopersicum L. Ciencia \& Tecnología Agropecuaria, 21(3). https://doi.org/10.21930/ rcta.vol21_num3_art:1344

Salazar, S. A., Quintero, Rojas, J. (2020b). Optimization of the tetrazolium test in three species of orchids of the Andean forest. Australian Journal Crop Sci, 14(5), 822-830. https://doi.org/10.21475/ajcs.20.14.05.p2276

Salazar-Mercado, S., Quintero-Caleño, Q., \& Rojas-Suárez, J. (2020) Determinación de la viabilidad de semillas de Capsicum annuum L. utilizando la prueba de tetrazolio. Aibi Revista Investig Adm Ing., 8(3), 7-12. https://doi. org/10.15649/2346030X.718

Serraj, R., McNally, K., Slamet, I., Kohli, A., Haefele, S., Atlin, G., \& Kumar, A. (2011). Drought resistance improvement in rice: An integrated genetic and resource management strategy. Plant Production Science, 14(1), 1-14. https://doi.org/10.1626/ pps.14.1 
Shayganya, J., Peivandya, N., \& Ghasemi, S. (2012). Increased yield of direct seeded rice (Oryza sativa L.) by foliar fertilization through multi-component fertilizers. Archives of Agronomy and Soil Science, 58(10), 1091-1098. https://doi.org/10.1080/0365 0340.2011 .570336

Siddika, M., Abedin, M., Sharmin, T., Hanif, M., \& Chandra, P. (2016). Effect of different micronutrients on growth and yield of rice. International Journal of Plant and Soil Science, 12(6), 1-8. https://doi.org/10.9734/ IJPSS/2016/28707

Siebenmorgen, T. (2013). Laboratory measurement of rice milling yield. En J. T. Hardke (ed.), Rice production handbook (pp. 167-173). University of Arkansas.

Virgili, A. (1996). Introducción a la fertilización con microelementos. Welgro Micromix, 1(2), 3-10.

Yamasaki, H., Pilon, M., \& Shikanai, T. (2008). How do plants respond to copper deficiency?. Plant Signaling \& Behavior, 3(4), 231-232. https://doi. org/10.4161/psb.3.4.5094

Yuan, L., Wu, L., Yang, C., \& Lv, Q. (2012). Effects of iron and zinc foliar applications on rice plants and their grain accumulation and grain nutritional quality. J Sci Food Agric., 93(2), 254-261. https:// doi.org/10.1002/jsfa.5749

Yruela, I. (2005). Copper in plants. Braziliam Journal of Plant Physiology, 17(1), 145-156. https://doi. org/10.1590/S1677-04202005000100012

Zhang, C., Zhao, W., Gao, A., Su, T., Wang, Y., Zhang, Y., Zhou, X., \& He, X. (2017). How could agronomic biofortification of rice be an alternative strategy with higher cost-effectiveness for human iron and zinc deficiency in China?. Food and Nutrition Bulletin, 39(2), 246-259. https://doi.org/10.1177/0379572117745661 